\title{
SOURCES AND BIASES OF THE PREDICTORS OF THE PROPERTIES OF RECOMBINANT INBREDS PRODUCED BY SINGLE SEED DESCENT
}

\author{
H. S. POONI and J. L. JINKS \\ Department of Genetics, University of Birmingham, Birmingham B15 2TT, England
}

Received 5.vi.78

\begin{abstract}
SUMmary
Four sources of estimates of the additive genetic component of variance, whose square root $(\sqrt{ } D)$ is one of the three parameters used to predict the properties of random inbreds derived from an $F_{2}$ by single seed descent are compared theoretically and experimentally using estimates from two Nicotiana rustica experiments. The comparisons are mainly based on the level of agreement between $\sqrt{ } D$ estimated from $F_{2}$ and first backcross $\left(B_{1}\right.$ and $\left.B_{2}\right)$ families and the $L_{1 i}\left(F_{2} \times P_{1}\right), L_{2 i}\left(F_{2} \times P_{2}\right)$ and $L_{3 i}\left(F_{2} \times F_{1}\right)$ families of an $F_{2}$ triple test cross and that observed among the random inbreds and to a lesser extent on the magnitudes of the standard errors of the estimates.

On level of agreement, the estimates from all sources were equally good in the absence of non-allelic interaction. In its presence those from the $F_{2}, B_{1}$ and $\mathrm{B}_{2}$ families were always among the worst. On balance the estimates from $\left(L_{1} i+L_{2} i\right)$ families were best in the presence of duplicate interactions, those from $\left(\mathrm{L}_{1 i}+\mathrm{L}_{2 i}+\mathrm{L}_{3 i}\right)$ families were best in the presence of complimentary interactions and those from $\mathrm{L}_{3}$ families were best with a mixture of the two kinds of interaction. For estimates based on the same total number of individuals those from $\left(\mathrm{L}_{1 i}+\mathrm{L}_{2 i}\right)$ families had the smallest and those from $\mathrm{F}_{2}$, $B_{1}$ and $B_{2}$ and $L_{3 i}$ families the largest sampling errors in most circumstances.
\end{abstract}

\section{InTRODUGTION}

IN previous papers (Jinks and Pooni, 1976; Pooni, Jinks and Cornish, 1977; and Pooni and Jinks, 1978) we have shown that the properties of the recombinant inbred lines derivable by single seed descent from an $F_{2}$ of a cross between two pure-breeding lines can be predicted from estimates of three genetical parameters; additive genetic $[d]$, and additive $\times$ additive interaction [i] components of family means and the square root of the additive genetic component of variation $D$ (see Mather and Jinks, 1971). Initially we obtained these estimates from a variety of sources that were available to us. For example, we used the first generations of selfing $\left(F_{2}\right)$ and backcrossing $\left(B_{1}\right.$ and $\left.B_{2}\right)$ following an initial cross $\left(F_{1}\right)$ between two pure-breeding lines $\left(\mathrm{P}_{1}\right.$ and $\mathrm{P}_{2}$ ) sometimes supplemented by more advanced generations. We also used the $\mathrm{L}_{1 i}\left(\mathrm{~F}_{2} \times \mathrm{P}_{1}\right), \mathrm{L}_{2 i}\left(\mathrm{~F}_{2} \times \mathrm{P}_{2}\right)$ and $\mathrm{L}_{3 i}\left(\mathrm{~F}_{2} \times \mathrm{F}_{1}\right)$ families of a triple test cross. But while all of these sources were more or less satisfactory in practice, our more recent predictions have been based upon the ideal combination of weighted least squares estimates of $[d]$ and $[i]$ obtained from the means of the basic generations $\left(\mathrm{P}_{1}, \mathrm{P}_{2}, \mathrm{~F}_{1}, \mathrm{~F}_{2}, \mathrm{~B}_{1}\right.$ and $\left.\mathrm{B}_{2}\right)$ and an estimate of $\sqrt{D}$ from the $\left(\mathrm{L}_{1 i}+\mathrm{L}_{2 i}+\mathrm{L}_{3 i}\right)$ comparison of a triple test cross. Estimates from these sources have led to reliable predictions over a 
wide range of circumstances including the presence of biases due to nonallelic interactions (Pooni, Jinks and Jayasekara, 1978; Pooni, Jinks and Cornish, 1977).

The crossing programme required to obtain these estimates, however, is relatively demanding and it is appropriate to ask whether the simpler and smaller crossing programme that make up the ideal combination would allow us to make predictions with an acceptable level of precision over a comparatively wide range of circumstances. In this paper we therefore compare theoretically and experimentally four different sources of estimating $\sqrt{D}$ within the basic generations and families of a triple test cross. For each source we derive the theoretical biases of these estimates in the presence of non-allelic interactions and compute their numerical values for a range of genetical situations. In addition, experimental data from two Nicotiana rustica breeding programmes, one conducted during 1965 and the other in 1973 , are used to estimate $\sqrt{ } \bar{D}$ from each of these sources for comparison.

\section{Genetical expectations}

(i) Non-allelic interactions absent

Where an additive genetic $(D)$, dominance $(H)$ and additive environmental $(E)$ model is adequate to account for all the variation, a random set of inbred lines derived by single seed descent from an $\mathrm{F}_{2}$ of a cross between two pure breeding lines is expected to display a genetic variance equal to $D$. In these circumstances the $\sigma^{2}$ 's obtained from the $\left(\mathrm{L}_{1 i}+\mathrm{L}_{2 i}+\mathrm{L}_{3 i}\right)$, $\left(\mathrm{L}_{1 i}+\mathrm{L}_{2 i}\right)$ and $\mathrm{L}_{3 i}$ comparisons among the triple test cross families will all provide a direct and unbiased estimate of $\frac{1}{8} D$. Similarly, a weighted least squares estimate of $D$ can be obtained from the basic generations. If the additive-dominance model is adequate and all the parameters in the model are significant this estimate of $D$ will not differ from that obtained directly from the variances of $\mathrm{F}_{2}, \mathrm{~B}_{1}$ and $\mathrm{B}_{2}$ families $\left(\mathrm{VF}_{2}, \mathrm{VB}_{1}\right.$ and $\mathrm{VB}_{2}$ respectively) as $D=4 \mathrm{VF}_{2}-2 \mathrm{VB}_{1}-2 \mathrm{VB}_{2}$. The value of $\sqrt{D}$ is directly obtained as the square root of any of these estimates.

\section{(ii) Non-allelic interactions present}

In the presence of non-allelic interactions the expected genetic variance of the random inbreds changes from $D=\sum d_{j}^{2}$ to $\sum d_{j}^{2}+\sum i_{j k}^{2}$ where $i_{f k}$ is the additive $\times$ additive interaction between the $j$ th and $k$ th locus as defined by Mather and Jinks (1977). Expectations of $\mathrm{L}_{1 i}, \mathrm{~L}_{2 i}$ and $\mathrm{L}_{3 i}$ family means for a digenic model with epistatic effects have been described by Kearsey and Jinks (1968). The corresponding $\left(\mathrm{L}_{1 i}+\mathrm{L}_{2 i}+\mathrm{L}_{3 i}\right)$ and $\left(\mathrm{L}_{1 i}+\mathrm{L}_{2 i}\right)$ comparisons have been derived by Pooni (1976) who also extended these digenic models to cover multi-locus situations. Mather and Jinks (1977) give the expected $F_{2}, B_{1}$ and $B_{2}$ variances for digenic interactions and these have also been extended to multi-locus situations.

The genetical expectations of the estimates of the additive variance that could be obtained from the $\left(\mathrm{L}_{1 i}+\mathrm{L}_{2 i}+\mathrm{L}_{3 i}\right),\left(\mathrm{L}_{1 i}+\mathrm{L}_{2 i}\right), \mathrm{L}_{3 i}$ and $\left(4 \mathrm{VF}_{2}-\right.$ $2 \mathrm{VB}_{1}-2 \mathrm{VB}_{2}$ ) comparisons in the presence of non-allelic interactions are tabulated in table 1 along with the expected genetic variance of the randomly derived inbreds. The estimate of $\sqrt{D}$ under the present circumstances will again be the square root of these rather complex expectations. 
TABLE 1

Genetical expectations of additive effects (D) when estimated from the random inbreds, $\left(L_{11}+L_{21}+L_{31}\right)$, $\left(L_{11}+L_{21}\right),\left(L_{3 \mathrm{i}}\right)$ and $\left(4 V F_{2}-2 V B_{1}-2 V B_{2}\right)$ comparisons in the presence of epistasis

Comparison

Random inbreds

$\left(\mathrm{L}_{1 i}+\mathrm{L}_{2 i}+\mathrm{L}_{3 i}\right)$

$\left(\mathrm{L}_{1 i}+\mathrm{L}_{2 i}\right)$

$\left(\mathrm{L}_{3 i}\right)$

$\left(4 \mathrm{VF}_{1}-2 \mathrm{VB}_{1}-2 \mathrm{VB}_{2}\right)$
Expectation

$\Sigma d_{j}^{2}+\Sigma i_{j k}^{2}$

$\Sigma d_{j}^{2}+\frac{1}{4} \sum i_{j k}^{2}+\frac{13}{18} \Sigma j_{j k}^{2}+\frac{1}{8} \Sigma 1_{j k}^{2}+\Sigma d_{j} \Sigma j_{j k}^{*}$

$-\frac{2}{3}\left[\Sigma d_{j} \sum j_{k j}\right]^{*}+\frac{1}{3}\left[\Sigma i_{j k} 1_{j k}\right]+\frac{2}{3}\left[\Sigma j_{j k j} j_{k j}\right]+\frac{1}{2} \Sigma j_{j k} \Sigma j_{j s}$

$+\left[\sum j_{j k}\left(\frac{2}{9} \Sigma j_{s k}-\frac{1}{3} \sum j_{k s}-\frac{1}{3} \Sigma j_{s j}\right)\right]$

$\Sigma d_{j}^{2}+\frac{1}{8} \sum i_{j k}^{2}+\frac{1}{8} \Sigma 1_{j k}^{2}+\frac{1}{2} \Sigma j_{j k}^{2}+\sum d_{j} \Sigma j_{j k}^{*}$

$-\left[\Sigma d_{j} \Sigma j_{k j}\right]^{*}+\frac{1}{4}\left[\Sigma i_{j k} l_{j k}\right]-\left[\Sigma j_{j k} j_{k j}\right]$

$+\frac{1}{2} \Sigma j_{j k} \sum j_{j s}+\frac{1}{2}\left[\Sigma j_{j k}\left(\Sigma j_{s k}-\Sigma j_{s j}-\Sigma j j_{k s}\right)\right]$

$$
\Sigma d_{j}^{2}+\frac{1}{8} \sum i_{j k}^{2}+\frac{1}{4} \Sigma j_{j k}^{2}+\Sigma d_{j} \Sigma j_{j k}^{*}+\frac{1}{2} \Sigma j_{j k} \Sigma j_{j s}
$$

$\Sigma d_{j}^{2}+\frac{1}{4} \Sigma i_{j k}^{2}+\frac{1}{4} \Sigma j_{j k}^{2}+\Sigma d_{j} \Sigma j_{j k}{ }^{*}+\left[\Sigma d_{j} \Sigma j_{j k}\right]^{*}$

$+\left[\Sigma i_{j k}\left(h_{j}+h_{k}\right)\right]^{*}-\frac{1}{2} \sum i_{j k}\left(\sum i_{j s}+\sum i_{k s}\right)+\frac{1}{2}[i] \Sigma 1_{j k}$

$+\frac{1}{2} \Sigma j_{j k} \Sigma j_{j s}-\frac{1}{2}\left[\Sigma j_{j k j} j_{k j}\right]+\frac{1}{2}\left[\Sigma j_{j k}\left(\Sigma j_{s k}+\Sigma j_{k s}+\sum j_{\delta j}\right)\right]$

The asterisked co-products are likely to change sign with the type of epistasis present and the bracketed ([ ]) expectations are dependent on the degree of gene association.

\section{Theoretical estimates}

It is quite evident from table 1 that the various estimates of $D$ are differentially biased by the epistatic effects so that they all differ from one another and from the value we are trying to predict, namely, the genetic variance of the random inbred lines. These expectations, however, are too complex for a visual judgment to be made about the relative magnitudes and directions of the biases. One way of proceeding is to compare these estimates for a number of different genetical situations by allocating a range of relative numerical values to the individual components of the expectations.

The estimates of $D$ were therefore computed from each of these expectations for the following levels of dominance ratio and coefficients of gene association.

Dominance ratio $=(H / D)^{\frac{1}{2}}=0.00,0.25,0.50,0.75$ and $1 \cdot 00$

Gene association $=r=0.00,0.20,0.40,0.60,0.80$ and 1.00

Segregation is assumed to take place at 10 independent loci with an additive genetic variance $D=\Sigma d_{j}^{2}=200$ and the allelic and non-allelic effects are defined as follows: The 10 loci are divided into two groups of five so that the type of epistasis displayed by each group could be specified independently as either complimentary or duplicate by making the epistatic effects positive or negative, respectively (Mather and Jinks, 1971). Following Pooni and Jinks (1976) the additive $\times$ additive $\left(i_{j k}\right)$, additive $\times$ dominance $\left(j_{j k}\right)$ and dominance $\times$ dominance $\left(l_{j k}\right)$ epistatic components for the $j$ th and $k$ th locus were allowed to take two values relative to the values of $D$ and $H$, by allowing $f$ to equal 0.1 or 0.2 in the expressions $(f . D / 40)^{\frac{1}{2}}$, $(f(D+H) / 80)^{\frac{1}{2}}$ and $(f . H / 40)^{\frac{1}{2}}$, respectively. 


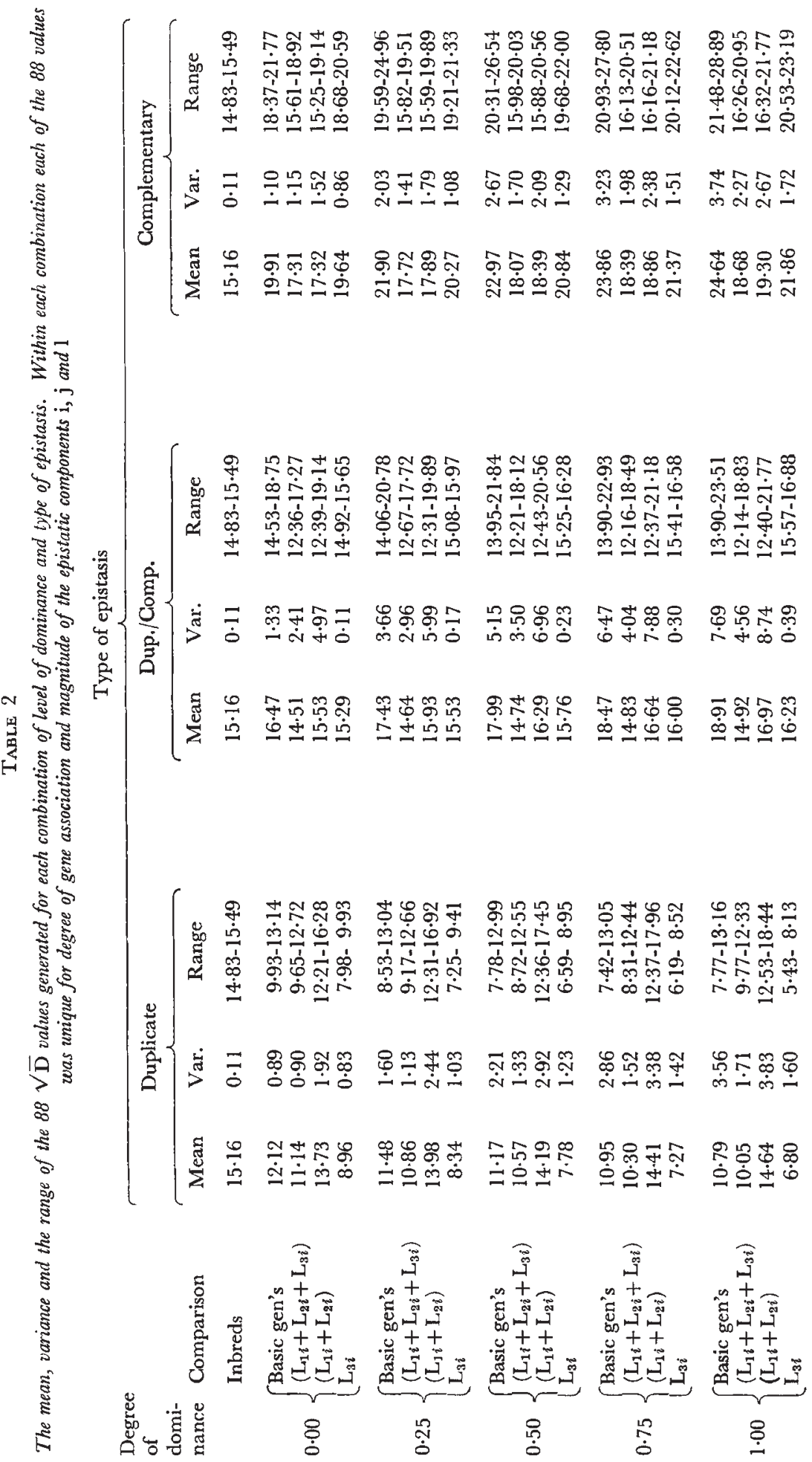


Each level of gene association with the exception of complete association $(r=1.00)$ can be generated in many different ways. For example, there are six ways of distributing the increasing and decreasing alleles between the two groups of five loci to achieve no association $(r=0.00)$ over all 10 loci. We could, of course, have included each of the possible ways of generating each of the six levels of association in our theoretical study. We chose, however, to cover the range of possibilities by including only the two extreme combinations for five of the levels of association $(r=0.00$ to 0.80$)$ along with the single combination which produces complete association $(r=1 \cdot 00)$. This leaves us, therefore, with 11 estimates of $D$ for the six levels of association for each of the five dominance ratios $\left((H / D)^{\frac{1}{2}}=0.00\right.$ to $1 \cdot 00)$, three types of epistasis (complementary, duplicate and mixture of half-complementary and half-duplicate) and eight combinations of levels of epistasis (each of two levels, $f=0.1$ and 0.2 ) for each of the three components.

In all 1320 estimates of $D$ were computed for each of the four sources of estimates. Of the four variables within each source, dominance ratio and type of epistasis had a much greater effect on the estimates than level of association and level of epistasis. For the purposes of presentation in table 2 we have, therefore, given the mean estimate of $D$ for each of the $15 \mathrm{com}$ binations of dominance ratio and type of epistasis for each of the four sources of estimates after averaging over levels of association and of epistasis. The lesser effects of levels of association and of epistasis on the estimates of $D$ are reflected in the variances of the estimates and the range, both of which are given alongside each mean estimate of $D$ in table 2. The mean, variance and range of each entry in the table is, therefore, based upon 88 estimates of $D$. Before discussing these results we shall describe some corresponding experimental results.

\section{EXPERIMENTAL ESTIMATES}

Data from two Nicotiana rustica breeding programmes have been analysed to obtain experimental estimates of the additive genetic statistic, $D$. The first experiment was conducted during the summer of 1965 and included $P_{1}, P_{2}, F_{1}, F_{2}, B_{1}$ and $B_{2}$. generations of the $V_{1} \times V_{5}$ cross together with $40 \mathrm{~L}_{1 i}, \mathrm{~L}_{2 i}$ and $\mathrm{L}_{3 i}$ families of a triple test cross produced by crossing each of 40 random $F_{2}$ individuals to $V_{1}, V_{5}$ and their $F_{1}$. The material was individually randomised within two replicate blocks and each plant was scored for final height and flowering time only. Further details of the experiment are given by Jinks and Perkins (1970) and Perkins and Jinks (1970).

In the second breeding programme $80 \mathrm{~F}_{11}$ inbreds derived by single seed descent from the $F_{2}$ individuals of the same $V_{1} \times V_{5}$ cross were raised as a part of a much larger experiment conducted during the summer of 1973. Five individuals from each of these families were individually randomised in each of the two replicate blocks and the material was scored for some 15 morphological and seed characters (Pooni, Jinks and Jayasekara, 1978). The results for flowering time and final height only will be considered here.

The data from the first experiment were used to provide predictor 
estimates of $\sqrt{D}$ while the observed $\sqrt{D}$ was obtained from the second experiment for both of the characters. These values of $\sqrt{D}$ are tabulated in table 3.

\section{TABLE 3}

Estimates of the predictor $\sqrt{\mathrm{D}}$ for flowering time and final height obtained from the basic generations $\left(P_{1}, P_{2}, F_{1}, F_{2}, B_{1}\right.$ and $\left.B_{2}\right)$ and the $\left(L_{1 \mathrm{i}}+L_{21}+L_{3 \mathrm{i}}\right),\left(L_{1 \mathrm{i}}+L_{2 \mathrm{i}}\right)$ and $L_{3 \mathrm{i}}$ families of a triple test cross of the $V_{1} \times V_{5}$ cross of Nicotiana rustica raised in 1965 and the value of $\sqrt{\bar{D}}$ observed in 1973 amongst a random sample of 80 inbred lines derived by single seed descent from the $F_{2}$ of the same cross

$\begin{array}{lcc} & \text { Flowering time } & \text { Final height } \\ \text { Source } & \sqrt{ } \bar{D} & \sqrt{ } \bar{D} \\ \text { Random inbreds } & 4 \cdot 80 & 13 \cdot 79 \\ \text { Basic generations } & 4 \cdot 33 & 13 \cdot 86 \\ \left(\mathrm{~L}_{11}+\mathrm{L}_{21}+\mathrm{L}_{3 \mathrm{i}}\right) & 5 \cdot 09 & 14 \cdot 07 \\ \left(\mathrm{~L}_{11}+\mathrm{L}_{2 \mathrm{i}}\right) & 3 \cdot 99 & 13.58 \\ \mathrm{~L}_{3 \mathrm{i}} & 6 \cdot 05 & 15 \cdot 37\end{array}$

\section{Discussion and gonclusions}

The estimates of $\sqrt{D}$ from the four different sources can be assessed for their relative reliabilities as predictors on two criteria. The first and most obvious is the closeness of the agreement between the estimate of $\sqrt{D}$ and the same statistic observed among the random inbred lines produced by single seed descent. On this criterion all four sources of estimates are equally reliable in the absence of non-allelic interactions. This, however, is no longer true if we consider the second criterion, namely, their sampling errors which will differ irrespective of the presence or absence of non-allelic interactions. For example, because of the inherently greater variation due to genetic segregation in the $\mathrm{L}_{3 i}$ as opposed to the $\mathrm{L}_{1 i}$ and $\mathrm{L}_{2 i}$ families of a triple test cross (Kearsey and Jinks, 1968) any estimate of $\sqrt{D}$ based upon comparisons which include $\mathrm{L}_{3 i}$ families will have a larger sampling error. For comparisons based upon the same total number of observations, therefore, the most reliable of the three estimates that can be obtained from the families of a triple test cross will be that based on the $\left(\mathrm{L}_{1 i}+\mathrm{L}_{2 i}\right)$ comparison and the least reliable that based on the $\mathrm{L}_{3 i}$ families. This of course will be true for most genetical situations except those with relatively low heritability values.

For the estimate of $\sqrt{D}$ derived from the $F_{2}$ and backcross families an additional factor, the non-independence of the estimates of the four parameters $D, H, F$ and $E$ (Mather and Jinks, 1971) reduces the relative reliability even further.

In the presence of non-allelic interactions the four estimates of $\sqrt{D}$ and the corresponding statistic for the inbred lines derived by single seed descent have different theoretical expectations (table 1), different numerical values (table 2) and different observed values (table 3). They differ in reliability, therefore, on the first as well as on the second criterion.

In the presence of non-allelic interactions the value of $D$ among the random inbred lines will be inflated by an amount equal to the additive $\times$ additive interaction variance $\sum i_{j k}^{2}$ (table 1 ). In contrast, the additive $\times$ 
dominance interaction is a major source of bias in all four sources of estimates of $\sqrt{D}$ and its magnitude depends on a combination of the direction of dominance and the type of interaction, complementary or duplicate, and the degree of gene association in the original parental inbreds $\left(\mathbf{P}_{1}\right.$ and $\left.\mathbf{P}_{2}\right)$.

Reference to table 2 shows that on our first criterion there is no one source that invariably provides the most reliable estimate of $\sqrt{D}$. The estimate from the $\mathrm{F}_{2}$ and backcross families, however, is always among. the least satisfactory and in most circumstances it is clearly the least reliable of the four sources. Each of the other three sources, however, provides the best estimate over a limited range of conditions. A clear example is the estimate from $\mathrm{L}_{3 i}$ families which in the presence of a mixture of complementary and duplicate interactions is the most reliable for all degrees of dominance, levels of interaction and degrees of association. In complete contrast the same source provides some of the poorest estimates when all the interactions are of the duplicate kind. While other examples are less striking, the best source of estimates in the presence of duplicate interactions is on balance $\left(\mathrm{L}_{1 i}+\mathrm{L}_{2 i}\right)$ and in the presence of complementary interactions, $\left(\mathrm{L}_{1 i}+\mathrm{L}_{2 i}+\mathrm{L}_{3 i}\right)$. Even in the presence of duplicate interactions, however, the estimates from $\left(\mathrm{L}_{1 i}+\mathrm{L}_{2 i}+\mathrm{L}_{3 i}\right)$ always fall between those from the other two sources. While therefore, they are not the best estimates in these conditions, they are also never among the worst.

The estimates obtained from the $\mathrm{L}_{3 i}$ families, on the other hand, are the most sensitive of the three to changes in the type of non-allelic interactions present while being relatively insensitive to changes in levels of dominance and interaction and degrees of association. These estimates therefore inform us about the type of epistasis present. For example, reference to Table 2 shows that if the $\mathrm{L}_{3 i}$ families yield the lowest estimate of $\sqrt{D}$ the interactions are predominantly duplicate while if they yield the highest estimate they are predominantly complementary, irrespective of all other circumstances.

By raising a full triple test cross and estimating $D$ from all three sources we should be able to diagnose the predominant type of interaction and hence decide which of the three sources gives the best estimate for predictive purposes. For instance, if the estimate from the $\mathrm{L}_{3 i}$ is the smallest the interaction is presumably predominantly of the duplicate type and the best estimate of $\sqrt{D}$ in these circumstances is provided by the $\left(\mathrm{L}_{1 i}+\mathrm{L}_{2 i}\right)$ comparison. On the other hand, if the estimate from $\mathrm{L}_{3 i}$ is the largest then by the same argument the best estimate is obtained from $\left(\mathrm{L}_{1 i}+\mathrm{L}_{2 i}+\mathrm{L}_{3 i}\right)$.

In extrapolating these conclusions to experimental situations two further points must be borne in mind. Firstly, the levels of interaction $(f=0 \cdot 1$ and $0 \cdot 2$ ) assumed in the numerical values in table 2 are higher than are usually encountered in practice and hence they over-emphasise the differences that we might expect to find between estimates from the different sources. Secondly, estimates from the different sources will have different sampling errors for the reasons discussed earlier. For a fixed number of individuals, therefore, the estimate based on the $\left(\mathrm{L}_{1 i}+\mathrm{L}_{2 i}\right)$ comparison will be the most reliable and that based on $\mathrm{L}_{3 i}$ families the least reliable. To achieve comparable reliabilities a greater number of $\mathrm{L}_{3 i}$ families and sibs per family must be raised.

The experimental results in table 3 are in general agreement with 
these theoretical expectations although they differ from them in detail. For final height there was no evidence of non-allelic interactions in the 1965 experiment and as expected all the estimates of $\sqrt{D}$ are in good agreement with one another and with the same statistic derived from the random inbreds produced by single seed descent (Jinks and Perkins, 1970). Not surprisingly, therefore, all sources of estimates of the predictor $\sqrt{D}$ provide good predictions of the properties of the inbred lines in respect of final height (Jinks and Pooni, 1976, Pooni, Jinks and Cornish, 1977).

For flowering time, on the other hand, there was evidence of significant non-allelic interactions in 1965 and as expected there are somewhat greater differences among the four estimates of the predictor $\sqrt{\bar{D}}$. The best estimate of the latter is clearly that from $\left(\mathrm{L}_{1 i}+\mathrm{L}_{2 i}+\mathrm{L}_{3 i}\right)$. Relative to this, the estimate from the $\mathrm{L}_{3 i}$ families is inflated and those from $\left(\mathrm{L}_{1 i}+\mathrm{L}_{2 i}\right)$ and the $F_{2}$ and backcross families deflated, which is also the pattern shown by the four estimates for final height. It is not, however, a pattern shown by any of the models in table 2 . This suggests that the differences arise as much from sampling error as from the relatively low level of non-allelic interactions in the $\mathrm{V}_{1} \times \mathrm{V}_{5}$ cross.

Clearer evidence of the effects of non-allelic interactions on estimates of $\sqrt{D}$ from the four sources must await the analysis of comparable experiments based on a cross between $\mathrm{V}_{2}$ and $\mathrm{V}_{12}$ of our Nicotiana rustica collection.

\section{REFERENCES}

JINKs, J. L., AND PERKINS, J. M. 1970. A general method for the detection of additive, dominance and epistatic components of variation. III. $\mathbf{F}_{\mathbf{2}}$ and backcross populations. Heredity, 25, 419-429.

JINKS, J. L., AND POONI, H. S. 1976. Predicting the properties of recombinant inbred lines derived by single seed descent. Heredity, 36, 253-266.

KEARSEY, M. J., AND JiNks, J. L. 1968. A general method of detecting additive, dominance and epistatic variation for metrical traits. I. Theory, Heredity, 23, 403-409.

MAther, K., AND JINks, J. L. 1971. Biometrical Genetics. Chapman and Hall, London.

MATHER, K., AND JINKs, J. L. 1977. Introduction to Biometrical Genetics. Chapman and Hall, London.

PERKINS, J. M., AND JINKs, J. L. 1970. Detection and estimation of genotype-environmental, linkage and epistatic components of variation for a metrical trait. Heredity, 25, 157-177.

POONI, H. s. 1976. Exploitation of inbreds in Nicotiana rustica. Ph.D. thesis, Department of Genetics, University of Birmingham.

POONI, H. s., AND JINKS, J. L. 1976. The efficiency and optimal size of triple test cross designs for detecting epistatic variation. Heredity, 36, 215-227.

POONI, H. S., AND JINKS, J. L. 1978. Predicting the properties of recombinant inbred lines derived by single seed descent for two or more characters simultaneously. Heredity, 40 , 349-361.

POONI, H. s., JINKS, J. L., AND CORNISH, M. A. 1977. The causes and consequences of nonnormality in predicting properties of recombinant inbred lines. Heredity, 38, 329-338.

POONI, H. S., JiNKs, J. L., AND JAYASEKARA, N. E. M. 1978. An investigation of gene action and genotype $\times$ environment interaction in two crosses of Nicotiana rustica by triple test cross and inbred line analysis. Heredity, 41, 83-92. 